\title{
Associative symmetry: VI. The effect of varying the interstimulus interval upon backward learning during classical conditioning
}

\author{
LEONARD BROSGOLE and FRED C. ANNICELLI \\ St. John's University, Jamaica, New York 11439
}

\begin{abstract}
A subthreshold tone was paired with a light that followed. The interval between the offset of the tone and the onset of the light was varied over groups. There was no interstimulus interval for Group 1, a .5-sec interval for Group 2, and a 1.0-sec interval for Group 3. It was found that the subsequent presentation of the light alone elicited the experience of a very low tone in the first two groups, but not in the third. It was concluded that the hallucinatory tone resulted from a backward connection formed during training. In addition, the strength of backward learning varied as a function of the interstimulus interval, just as in forward conditioning.
\end{abstract}

In an earlier study, Brosgole and Lefkowitz (1976) found evidence for backward learning during classical conditioning. Human observers formed a conditioned discrimination over a series of 100 trials. On 50 trials, a subliminal tone of 2 -sec duration was followed by a 2-sec flash of light (positive stimulus) that appeared $1 \mathrm{ft}$ to the left of center for half the subjects and $1 \mathrm{ft}$ to the right of center for the remaining half. The tone and light overlapped for $1 \mathrm{sec}$. On the remaining 50 trials, a 2-sec flash of light (negative stimulus) appeared in the absence of a tone. The positive and negative stimuli were presented in opposite locations, so that if the positive light was to the left for a given subject, the negative one was to the right. Thus, position was used to enable a discrimination to be formed, with the order of the two stimuli counterbalanced over trials. The subjects responded by depressing one of two push buttons upon the offset of the lights, only if they thought they heard a tone. They pressed a button to the left if the tone was heard prior to the light and a button to the right if the tone seemed to occasion the onset of the light. Accordingly, hits and misses were obtained for the positive light, while false alarms and correct rejections were gotten to the negative light.

Conditioning was followed by an additional 100 trials, where the presentation of the positive and negative stimuli was again counterbalanced. However, the tone was now omitted, thereby enabling false alarms to be obtained to each of the two stimuli. It was reasoned that if backward connections were being formed between the tone and positive stimulus during conditioning, then the positive light should have elicited a greater abundance of false alarms than the negative one. It was found that the subjects reported hearing a tone significantly more often to the positive light. In addition, the hallucinatory tonal experience predominantly occasioned the onset of the light and, indeed, seemed to be produced by the positive light itself. It was concluded that this phenomenon could have resulted only from backward learning.

It may be contended that, since the subliminal tone and positive stimulus overlapped in time, the original training may have resulted in two forward connections: one between tone and light and the other between light and tone. Thus, the conditioned hallucination may have resulted from forward, rather than backward, learning. The following study was directed at controlling for such an argument by temporally separating the tone and light during original training.

\section{METHOD}

Subjects

Twelve males and 12 females participated in this study. Their ages ranged from 19 to 27 years; their mean age was 21.5 years. All were naive as to the purpose of the experiment.

\section{General}

The logic of the experiment was simple, but its implementation was rather complicated. The first step involved obtaining the subjects' threshold for the tone used throughout the conditioning session. This was followed by 210 trials. In the first 100 trials, the tone was presented with the positive light 50 times, while the negative light appeared alone (with no tone) 50 times. The positive light was on the right for half the subjects and on the left for the other half. The remaining 110 trials consisted of 50 presentations of the positive light and 50 presentations of the negative light. The tone was omitted at all times. The order of presentation was counterbalanced so that each light appeared five times in 10-trial blocks. The remaining 10 trials consisted of reinforced presentations, where the tone and positive light were paired. One such trial was inserted randomly in each 10-trial block, in order to maintain performance. The subject was required to respond on all trials throughout the entire session by depressing telegraph keys if a tone was heard. Each trial was initiated by the presentation of an auditory warning signal, so as to maintain alertness in the rather long and boring procedure. Thresholds were determined in the conditioning setting itself. 


\section{Treatments}

There were three groups of eight subjects. For Group 1, the onset of the positive light was simultaneous with the offset of the subliminal tone. The interstimulus interval was .5 and $1.0 \mathrm{sec}$ for Groups 2 and 3, respectively.

\section{Apparatus}

Testing took place in a dimly lit Industrial Acoustics chamber (Model 1203A), with the subject seated at a table along a wall dividing the chamber from an adjoining control room. Communication between the chamber and control room was accomplished by use of a Fannon intercom system. The entire experimental sequence was controlled by a logic circuit composed of BRS digibit components (Series 200), along with some relay equipment. A $2,900-\mathrm{Hz}$ warning signal was produced by a BRS Sonalert unit (SA 201) which gave rise to a .1-sec chirp-like sound. The unit was located at the left rear corner of the table. The tone that followed was $900 \mathrm{~Hz}$. It was produced by a BRS audio oscillator (AO 201) and was fed through a BRS audio amplifier (AA 201) into a General Radio audio frequency microvolter (Model 546C) which was used to regulate the intensity of the tone. The output of the microvolter was fed into a Lafayette stereophonic headset worn by the subject. A vertical wooden panel, covered with black felt, was affixed to the table in front of the subject. Two wooden arms extended forward horizontally from the bottom of the panel. A telegraph key was mounted on each arm. They were 16 in. apart and served as the response keys by which the subject indicated the fashion in which the tone was experienced. Switch closures were displayed on electronic counters and recorded manually. Two lamp sockets with amber covers were mounted on the vertical panel. They were 24 in. apart laterally from center to center and contained $12-\mathrm{V}$ dc lamps which were powered by the BRS circuit. One was 12 in. to the left of the subject and the other 12 in. to the right. Both approximated the subjects' eye level.

\section{Procedure}

The subjects were told that this was a probability learning experiment where a subthreshold tone would be differentially paired with two lights. They were informed that the probabilities would vary over subjects, so that one person might always receive the tone to one light and not at all to the other, while the tone might be distributed equally between the lights for another individual. They were further told to be keenly aware of the lights, so that they would be in a position to estimate the relative probabilities at the conclusion of the procedure. They were further told to prepare themselves to listen for the tone upon hearing an audible chirp. Sometime after the chirp, a light would come on and go off. When the light went off, they were to indicate whether the tone was heard by pressing one of the two keys located in front of them-the key to the left if the tone was heard before the onset of the light, and the one to the right if the tone came on with or after the light. They were instructed to respond if they heard, felt, or in any way sensed the presence of the tone. No response was required if the tone was not heard. These instructions were specifically designed to drop the criterion level in a rather conservative subject population. However, the subjects were thoroughly informed about the intent of the study at the conclusion of the procedure.

Threshold was obtained for the $900-\mathrm{Hz}$ tone prior to the conditioning trials, using the method of limits. Fifteen ascending and 15 descending trials were counterbalanced. Each presentation of the tone was followed by the onset of both the positive and negative lights, with the subjects responding verbally through the intercom upon the offset of the lights. Threshold was established as the mean of the last five ascending and five descending trials. (The mean threshold over all subjects was $41.3 \mathrm{~dB}$, re: luv). The tone was then dropped by $3 \mathrm{~dB}$ at the commencement of conditioning and an additional $1.5 \mathrm{~dB} 10$ trials into the session. This was done to compensate for changes in sensitivity resulting from task-induced activation.

The threshold determination was immediately followed by the 100 trials of training. The order of presenting the positive and negative stimulus was counterbalanced, using the Fellows' series (1967). Every trial was initiated by the .1-sec warning signal. During all positive trials, the $900-\mathrm{Hz}$ tone followed the onset of the warning signal by $1.0 \mathrm{sec}$. The subliminal tone lasted for $2 \mathrm{sec}$. In Group 1, the offset of the tone was simultaneous with the onset of the positive light which lasted, as well, for $2 \mathrm{sec}$. Thus, during the other trials, where the tone was omitted, the negative light followed the onset of the warning signal by $3.0 \mathrm{sec}$. Similarly, the positive and negative lights followed the onset of the ready signal by 3.5 and $4.0 \mathrm{sec}$ in Groups 2 and 3 , respectively. There was always a $7.0-\mathrm{sec}$ intertrial interval. The timing sequence was the same during the latter portion of the procedure, where the subliminal tone was presented only upon occasion.

This design enabled the recording of false alarms to the positive and negative lights. It was reasoned that backward learning should have resulted in a greater false alarm frequency to the positive stimulus. By the use of such logic, then, the effect of varying the interstimulus interval upon a backward conditioned discrimination could be ascertained.

\section{RESULTS AND DISCUSSION}

The data were organized so as to be analyzed by a five-way analysis of variance. The first factor (A) was Groups, each group representing a different interstimulus interval. This was a between-subjects factor containing three levels $(0-, 0.5-$, and 1.0 -sec interval). The remaining four factors were all within-subject variables. Factor B contained two levels; the first 100 conditioning trials and the last 100 nonreinforced, or extinction, trials. The data were further organized in terms of the frequency of responding per every 10 presentations of each light in both conditioning and extinction (Factor C). Thus, Factor $\mathrm{C}$ provided a means of looking at conditioning and extinction as a function of passing trials. Accordingly, there were five successive blocks of 20 trials at each level of $B$, with each block of trials containing 10 presentations of the positive and negative lights. Factor D represented the type of response given, i. e., whether the tone was heard before the light appeared or if it seemed to be produced by the light itself. Thus, there were two levels of D. Finally, Factor E had two levels, namely, the frequency of responding to the positive and negative stimulus.

During conditioning, each group had the possibility of 400 hits to the positive stimulus and 400 false alarms to the negative light. That is to say, every subject had 100 opportunities to report a tone, 50 to each light. Similarly, there could have been 400 false alarms to each light during extinction. With regard to the positive stimulus, Group 1 afforded a total of 326 hits during conditioning, compared to 324 for Group 2 and 223 for Group 3. The question may be raised as to why Groups 1 and 2 were so far above threshold in reporting the presence of the tone. It must be noted that Group 1 reported the appearance of the tone with the onset of 
Table 1

Number of Responses to the Positive Stimulus by Groups 1-3 at Each Block of Testing During Conditioning and Extinction

\begin{tabular}{|c|c|c|c|c|c|c|c|c|c|c|c|c|}
\hline & \multicolumn{6}{|c|}{ Conditioning Block } & \multicolumn{6}{|c|}{ Extinction Block } \\
\hline & 1 & 2 & 3 & 4 & 5 & Total & 1 & 2 & 3 & 4 & 5 & Total \\
\hline & \multicolumn{12}{|c|}{ Group 1} \\
\hline Before Onset & 53 & 54 & 43 & 33 & 39 & 222 & 17 & 15 & 11 & 13 & 11 & 67 \\
\hline With or After Onset & 12 & 15 & 23 & 32 & 22 & 104 & 27 & 24 & 18 & 19 & 10 & 98 \\
\hline \multirow[t]{2}{*}{ Total } & 65 & 69 & 66 & 65 & 61 & 326 & 44 & 39 & 29 & 32 & 21 & 165 \\
\hline & \multicolumn{12}{|c|}{ Group 2} \\
\hline Before Onset & 53 & 40 & 44 & 45 & 39 & 221 & 17 & 19 & 14 & 6 & 10 & 66 \\
\hline With or After Onset & 13 & 24 & 23 & 21 & 22 & 193 & 18 & 24 & 24 & 25 & 16 & 110 \\
\hline Total & 66 & 64 & 67 & 66 & 61 & 324 & 36 & 43 & 38 & 32 & 27 & 176 \\
\hline & \multicolumn{12}{|c|}{ Group 3} \\
\hline Before Onset & 48 & 43 & 41 & 23 & 34 & 189 & 25 & 10 & 15 & 15 & 11 & 76 \\
\hline With or After Onset & 4 & 5 & 9 & 10 & 6 & 34 & 4 & 11 & 8 & 4 & 3 & 30 \\
\hline Total & 52 & 48 & 50 & 33 & 40 & 223 & 29 & 21 & 23 & 19 & 14 & 106 \\
\hline
\end{tabular}

the light on 104 occasions and Group 2 did so 103 times. Note that these alleged hits would have constituted clear misses were it not for the positive light. In other words, it was as if the positive light was generating false alarms, presumably by virtue of backward learning, so as to transform misses into hits. This phenomenon occurred only 34 times in Group 3. Thus, it would appear that the conditioning data reflect a marked weakening in backward learning with a 1.0 -sec interstimulus interval. Refer to Table 1.

The extinction data reflected the same differences between the grouns. Group 1 reported 165 false alarms, compared to 176 for Group 2 and 90 for Group 3. Of these, 98 were generated by the positive light in Group 1, 110 in Group 2, and only 30 in Group 3.

The negative stimulus was hardly as effective in differentiating the groups. Refer to Table 2. During conditioning, there were 69,44 , and 49 false alarms for Groups $1-3$, respectively. The light itself gererated 30 , 22 , and 24 false alarms for the three groups, in that order. During extinction there were totals of 102, 108, and 90 false alarms, with 43,51 , and 35 produced by the onset of the light. Thus, the two stimuli differed in their capacity to produce false alarms at a 0 - and .5 -sec interstimulus interval. This was not the case at $1.0 \mathrm{sec}$.

The data were subjected to a split-plot factorial analysis of variance with one between and four within factors, using the standard transformation of $\sqrt{\text { mean }+.5}$ for frequency scores. There was a significant five-way interaction $(\mathrm{F}=3.69, \mathrm{df}=8.84, \mathrm{p}<.01)$. Because of the extensive posttesting involved in such a high-order interaction, it is possible only to summarize the most salient aspects of the analysis.

Simple effects revealed that the onset of the positive light produced significantly more responses than the negative light in Group 1 over all blocks of trial during conditioning. The exposure of the positive light generated significantly more false alarms in Blocks 1,2 , and 4 of extinction. The two stimuli did not differ in Blocks 3 and 5 , indicating that the conditioned discrimination was being weakened by the drastic reduction in the number of reinforced trials. The same was the case for Group 2, except that the conditioned discrimination did not break down until the fifth block of the extinction trials. Finally, there was no difference between the

Table 2

Number of Responses to the Negative Stimulus by Groups 1-3 at Each Block of Testing During Conditioning and Extinction

\begin{tabular}{|c|c|c|c|c|c|c|c|c|c|c|c|c|}
\hline & \multicolumn{6}{|c|}{ Conditioning Block } & \multicolumn{6}{|c|}{ Extinction Block } \\
\hline & 1 & 2 & 3 & 4 & 5 & Total & 1 & 2 & 3 & 4 & 5 & Total \\
\hline & \multicolumn{12}{|c|}{ Group 1} \\
\hline Before Onset & 11 & 11 & 9 & 4 & 4 & 39 & 5 & 10 & 13 & 16 & 15 & 59 \\
\hline With or After Onset & 6 & 8 & 5 & 7 & 4 & 30 & 6 & 10 & 13 & 9 & 5 & 43 \\
\hline \multirow[t]{2}{*}{ Total } & 17 & 19 & 14 & 11 & 8 & 69 & 11 & 20 & 26 & 25 & 20 & 102 \\
\hline & \multicolumn{12}{|c|}{ Group 2} \\
\hline Before Onset & 3 & 6 & 3 & 4 & 6 & 22 & 8 & 8 & 14 & 15 & 12 & 57 \\
\hline With or After Onset & 2 & 5 & 7 & 4 & 4 & 22 & 5 & 12 & 11 & 11 & 12 & 51 \\
\hline \multirow[t]{2}{*}{ Total } & 5 & 11 & 10 & 8 & 10 & 44 & 13 & 20 & 25 & 26 & 24 & 108 \\
\hline & \multicolumn{12}{|c|}{ Group 3} \\
\hline Before Onset & 4 & 2 & 5 & 10 & 4 & 25 & 11 & 12 & 12 & 11 & 9 & 55 \\
\hline With or After Onset & 2 & 4 & 5 & 7 & 6 & 24 & 5 & 9 & 4 & 8 & 9 & 35 \\
\hline Total & 6 & 6 & 10 & 17 & 10 & 49 & 16 & 21 & 16 & 19 & 18 & 90 \\
\hline
\end{tabular}


stimuli at any point in conditioning and extinction for Group 3.

There is yet another way of looking at the data. The only systematic difference between the groups was the tendency for the positive stimulus to generate responses upon onset. It produced significantly more responses in Groups 1 and 2 from the very first block of conditioning trials through the second block of extinction trials. The groups were equivalent from the third block on, again reflecting a breakdown in the conditioned discrimination in Groups 1 and 2.

To summarize, the conditioning procedure led to a discrimination between the positive and negative stimuli at the 0 - and $.5-\mathrm{sec}$ interstimulus intervals. Apparently, this resulted in the formation of a bidirectional connection, evidenced by the tendency of the positive stimulus to elicit a hallucinatory tonal experience. This tendency extinguished as a function of nonreinforced trials.
The extinction data, coupled with a failure to produce backward learning at a $1.0-\mathrm{sec}$ interstimulus interval, fits the literature concerning forward connection. These results cannot be explained in terms of contiguous conditioning since a rather powerful effect was found with a .5 -sec interstimulus interval. In fact, it is interesting to note that Group 2 was most highly resistant to extinction. Accordingly, it may be concluded that bidirectional learning occurs during classical conditioning.

\section{REFERENCES}

Brosgole, L., \& Lefkowitz, S. J. Associative symmetry: IV. Classical conditioning in humans. Bulletin of the Psychonomic Society, 1976, 7, 173-176.

Fellows, B. J. Change in stimulus sequences for discrimination tasks. Psychological Bulletin, 1967, 67, 87-92.

(Received for publication May 20, 1976.) 